\title{
Cold intolerance in patients with angina pectoris: effect of nifedipine and propranolol
}

\author{
I PEART, R E BULLOCK, C ALBERS, R J C HALL \\ From the Department of Cardiology, Royal Victoria Infirmary, Newcastle upon Tyne
}

SUMMARY Fifteen patients with chronic stable angina pectoris and a history of reduced exercise tolerance in cold weather (cold intolerance) underwent symptom limited treadmill exercise tests at $20^{\circ} \mathrm{C}$ and $0^{\circ} \mathrm{C}$ in a specially constructed cold chamber while taking no antianginal medication. Their mean time to onset of angina (5.8v $4.2 \mathrm{~min})$, to $1 \mathrm{~mm} \mathrm{ST}$ depression (5.1 $v 3.8 \mathrm{~min})$, and to peak exercise $(7.4 v 5.7 \mathrm{~min})$ was significantly shorter on exercise at $0^{\circ} \mathrm{C}$ than at $20^{\circ} \mathrm{C}$. The double product of heart rate and systolic blood pressure at each of these end points was the same in both exercise tests. Eight of these patients were treated with nifedipine $10 \mathrm{mg}$ three times a day for two weeks and then with propranolol $40 \mathrm{mg}$ three times a day for another two weeks. Repeat exercise testing was performed at the end of each two week treatment period. The mean time (SD) to peak exercise at the end of the nifedipine treatment period was $9.1(2.0) \mathrm{min}$ at $20^{\circ} \mathrm{C}$ and $8.5(2.3) \mathrm{min}$ at $0^{\circ} \mathrm{C}$. The double product at peak exercise was the same for both exercise tests. At the end of the propranolol treatment period the mean time to peak exercise was significantly less at $0^{\circ} \mathrm{C}(7 \cdot 8(2 \cdot 6)$ min) than at $20^{\circ} \mathrm{C}(8.9(2.4) \mathrm{min})$. The double product at peak exercise was the same for both exercise tests but was significantly less than that on nifedipine.

Cold intolerance was shown in patients with a positive history by symptom limited treadmill exercise testing at $0^{\circ} \mathrm{C}$. It persisted when they were treated with propranolol, albeit to a lesser extent, but not when they were treated with nifedipine.

Not infrequently patients with chronic stable angina pectoris complain that their symptoms are more easily provoked in cold weather (cold intolerance). Although we found that $60 \%$ of patients with angina pectoris ${ }^{1}$ reported such cold intolerance it has proved very difficult to obtain objective evidence of this phenomenon. Indeed in one study only $50 \%$ of patients with a history of cold intolerance showed a decreased exercise tolerance in a cold environment. ${ }^{2}$

Various mechanisms have been suggested to explain the phenomenon, including a reflex increase in peripheral vascular resistance resulting in increased cardiac work, ${ }^{3}$ and a reflex increase in coronary artery tone or even coronary artery spasm producing a reduction in coronary perfusion. ${ }^{45}$

Cold intolerance is seldom considered when decisions are made about the treatment of patients with chronic stable angina. $\beta$ Blockers, ${ }^{6}$ for instance, increase both peripheral and coronary vascular resistance and could theoretically, therefore, worsen cold

Requests for reprints to Dr R J C Hall, Department of Cardiology, University Hospital of Wales, Heath Park, Cardiff CF4 4XW.

Accepted for publication 10 January 1989 intolerance. Calcium antagonists, ${ }^{7}$ on the other hand, reduce both peripheral and coronary vascular resistance and may, thus, be a better choice of antianginal agent in patients with cold intolerance.

The present study was designed to look at three problems. Is it possible to show by exercise testing at $0^{\circ} \mathrm{C}$ and $20^{\circ} \mathrm{C}$ the phenomenon of cold intolerance in a group of patients with chronic stable angina pectoris? Do $\beta$ blockers and calcium antagonists affect cold intolerance in different ways? Can simple observations of heart rate, blood pressure, and exercise tolerance shed light on the, at present, obscure mechanisms underlying the clinical phenomenon?

\section{Patients and methods}

\section{PATIENTS}

We studied 15 patients (four women and 11 men aged 40-60 (mean 49)) with a history of chronic stable angina pectoris who were not taking regular antianginal treatment apart from glyceryl trinitrate as required. All patients complained that their angina attacks were more frequent and more easily provoked in cold weather and at night. None had had an acute 
myocardial infarction in the preceding six months and none had undergone coronary artery surgery. Patients with chronic obstructive airways disease were excluded. Informed consent was obtained from all patients.

\section{EXERCISE TESTING}

Exercise testing was undertaken in a specially built room with internal insulation, a securely fitted refrigerator door and a duct through which cold air entered the room to cool the room before, and to maintain the temperature during, the cold exercise tests. The room was cooled by a continuous flow of cold air during the night before the cold exercise tests. This ensured that the wall temperature of the room was also at $0^{\circ} \mathrm{C}$. Exercise tests were performed at a room temperature of $18-20^{\circ} \mathrm{C}$ (warm exercise tests) and at $0^{\circ} \mathrm{C}$ (cold exercise tests). Temperatures were measured by thermocouples. During the cold exercise tests cold air was directed via the duct at the patient's face and trunk at a wind velocity of approximately $2 \mathrm{~m} / \mathrm{s}$. All monitoring and electrocardiographic recording equipment was moved out of the room for the cold exercise tests. During exercise testing these instruments were viewed through a window in the door. The treadmill was tested to ensure that there was no significant reduction in speed at $0^{\circ} \mathrm{C}$ compared with $20^{\circ} \mathrm{C}$. For the cold exercise tests the patients wore their normal shoes with trousers or a skirt, and a $T$ shirt covered by a warm, fibrepile anorak that was sleeveless to permit blood pressure recording.

All subjects underwent two preliminary treadmill exercise tests at room temperature $\left(18-20^{\circ} \mathrm{C}\right)$ using a modified Bruce protocol (table 1) on different days to acquaint them with the equipment and procedure to ensure that their exercise tolerance (under baseline conditions) was reproducible and that time to peak exercise did not vary by more than $5 \%$ between tests. Exercise tests (including those during the pharmacological study) were performed at the same time each day and the patients were asked to abstain from smoking and eating for at least four hours before the exercise test. Patients were asked not to take any glyceryl trinitrate on the day of their exercise tests. After they were attached to the electrocardiograph they rested for 15 minutes before any measurements were taken or the exercise test was started.

For the study, the warm and cold exercise tests were performed on consecutive mornings, the order having been randomised. Eight patients had their warm exercise test first and seven patients had their cold exercise test first.

A standard 12 lead electrocardiogram and blood pressure on a mercury random zero sphygmomanometer were recorded with the patient lying and
Table 1 Exercise protocol

\begin{tabular}{|c|c|c|}
\hline Stage & Speed (mph) & Gradient (\%) \\
\hline $\begin{array}{l}1 / 2 \\
1 \\
2 \\
3 \\
4 \\
5\end{array}$ & $\begin{array}{l}1.7 \\
1.7 \\
2.5 \\
3.4 \\
4.2 \\
5.0\end{array}$ & $\begin{array}{r}5 \\
10 \\
12 \\
14 \\
16 \\
18\end{array}$ \\
\hline
\end{tabular}

$1 \mathrm{mph}=1.6 \mathrm{k} / \mathrm{h}$.

standing at rest every minute during the exercise test, $\overrightarrow{\vec{\omega}}$ at peak exercise, and every minute after exercise until $\stackrel{\rho}{\circ}$ the electrocardiogram had returned to its resting? appearance. Leads II, V2, and V5 were recorded 9 continuously throughout all exercise tests. Facial, $\dot{\sigma}$ trunk, and forearm temperatures were monitored throughout the cold exercise tests. To exclude exer- $-\vec{T}$ cise induced asthma as a cause of symptoms a $\mathrm{S}$ Vitalograph was used to record forced expiratory $\vec{c}$ volume in one second and forced vital capacity both $\subseteq$ before and after exercise.

\section{PHARMACOLOGICAL INTERVENTION}

After we established that cold intolerance could be shown by the symptom limited exercise test at $0^{\circ} \mathrm{C}$ in the first seven patients, we treated the next eight patients (numbers 8-15 in table 3 and $1-8$ in table 5) with nifedipine and propranolol in a single blind but $\frac{\circ}{\circ}$ not randomised trial. We treated these eight patients $\stackrel{\varrho}{\rightarrow}$ for two weeks with nifedipine $10 \mathrm{mg}$ three times a day and repeated the warm $\left(20^{\circ} \mathrm{C}\right)$ and cold $\left(0^{\circ} \mathrm{C}\right)$ exercise tests at the end of the treatment period. Then we treated them with propranolol $(40 \mathrm{mg}$ three times a day) for a further two weeks and repeated the exercise tests.

DATA ANALYSIS

All exercise tests were symptom limited; before each $\stackrel{\delta}{0}$ test patients were asked to exercise until they felt that their angina was at a level that would normally cause $\frac{\rho}{5}$ them to stop during everyday activities. For each $D$ exercise test we noted the time of and the heart rate, blood pressure and double product (heart rate $\times N$ systolic blood pressure) at the onset of angina, the o development of $1 \mathrm{~mm}$ ST segment depression, and $N$ the termination of exercise. For the cold exercise $\omega$ tests the temperature of the cold room and each $₹$ patient's facial, trunk, and forearm temperatures atco peak exercise were recorded.

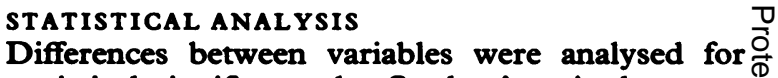
statistical significance by Student's paired $t$ tests. $\stackrel{\oplus}{\oplus}$ The results are presented as mean time and the $\stackrel{\mathbb{Q}}{\circ}$ standard error of the mean of the differences in $\bar{x}$ 
Table 2 Exercise test data (mean (SD)) for the 15 patients exercised at $0^{\circ} \mathrm{C}$ and $20^{\circ} \mathrm{C}$ on no antianginal medication

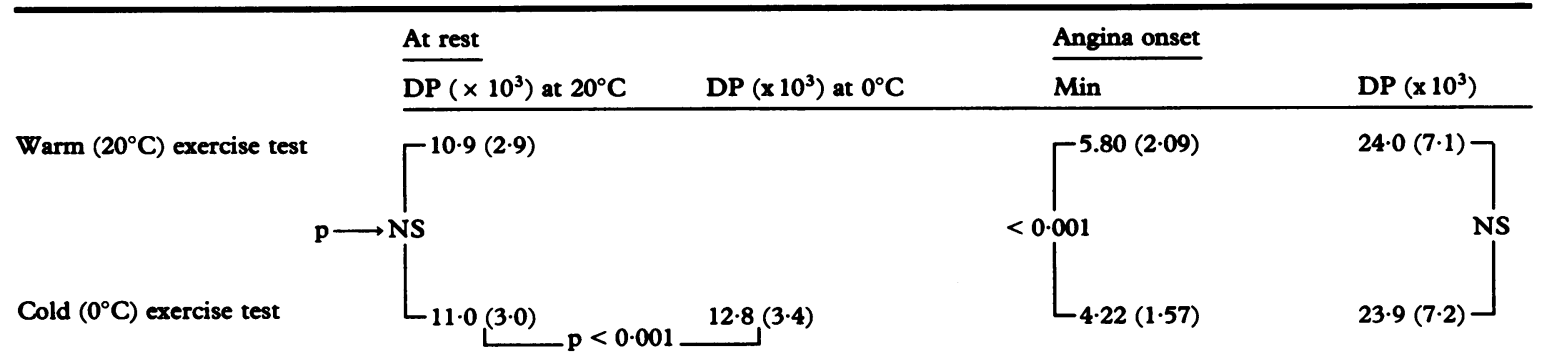

\begin{tabular}{|c|c|c|c|c|}
\hline & \multicolumn{2}{|c|}{$1 \mathrm{~mm} \mathrm{ST}$ depression } & Peak exercise & \multirow[b]{2}{*}{$\mathrm{DP}\left(\times 10^{3}\right)$} \\
\hline & Min & $\mathrm{DP}\left(\times 10^{3}\right)$ & Min & \\
\hline Warm $\left(20^{\circ} \mathrm{C}\right)$ exercise test & $\Gamma^{5 \cdot 14(2.33)}$ & $21.5(6.4)\urcorner$ & $\Gamma^{7.37(2.26)}$ & $26.1(8.0) \sqsupset$ \\
\hline & $<0.005$ & NS & 001 & NS \\
\hline Cold $\left(0^{\circ} \mathrm{C}\right)$ exercise test & $L_{3.77(1.58)}$ & $21.7(6.7)]$ & $L_{5.73(2.01)}$ & $25.9(7.5)$ \\
\hline
\end{tabular}

DP, double product of heart rate $\times$ blood pressure.

$\mathrm{DP}$ at $20^{\circ} \mathrm{C}$ for cold exercise test is DP before entry to cold room.

exercise time, with the $95 \%$ confidence interval. The data in the tables are means (SD).

\section{Results}

\section{NO TREATMENT}

Table 2 shows the group data for the 15 patients exercised at $0^{\circ} \mathrm{C}$ and $20^{\circ} \mathrm{C}$ on no antianginal medication. Table 3 and the figure show the data for individual patients. There was no difference in the resting double product at room temperature $\left(20^{\circ} \mathrm{C}\right)$ before either the warm or cold exercise tests $(10.9 \mathrm{v}$ $11 \cdot 0(0 \cdot 27), 95 \% \mathrm{CI}-0.48$ to $0 \cdot 68)$. After one minute in the cold room, before the cold exercise test, there was a significant increase in the double product (11.0 $v 12.8(0.23), 95 \%$ CI 1.31 to 2.29 ; p < 0.001$)$ but no change in the heart rate $(83.4 v 85.3(1.28)$ beats $/ \mathrm{min}$, $95 \%$ CI -0.85 to 4.65$)$. The important effect, therefore, was on the systolic blood pressure. The exercise time to onset of angina $(5.8 v \mathbf{4} .2(0.31) \mathrm{min}$, $95 \% \mathrm{CI} 0.94$ to $2 \cdot 26 ; 27.6 \%$ reduction; $p<0.001$ ), to the development of $1 \mathrm{~mm}$ ST depression $(5.1 v 3.8$ (0.32) $\mathrm{min}, 95 \%$ CI 0.61 to $2.0 ; 25.5 \%$ reduction; $p$ $<0.005)$, and to peak exercise $(7.4 v 5.7(0.24) \mathrm{min}$, $95 \% \mathrm{CI} 1 \cdot 19$ to $2 \cdot 21 ; 23.0 \%$ reduction; $\mathrm{p}<0.001$ ) all decreased significantly in the cold. The double product at each of these points was the same in the cold as in the warm although obviously it was reached more quickly.
There was no significant change in either forced expiratory volume in one second or forced vital capacity in any patient after any of the exercise tests.

\section{PHARMACOLOGICAL STUDY}

Tables 4 and 5 summarise the exercise test data obtained in the eight patients in the pharmacological study. Before treatment, cold intolerance had been shown in this group of patients. Their mean time to onset of angina $(5.4 v 3.5(0.47) \mathrm{min}, 95 \% \mathrm{CI} 0.79$ to 3.01; p < 0.01), to $1 \mathrm{~mm} \mathrm{ST} \mathrm{depression} \mathrm{(4.5} \mathrm{v} \mathrm{3.1}$ (0.44) $\min , 95 \%$ CI 0.36 to $2.44 ; \mathrm{p}<0.05$ ), and to peak exercise $(6.8 v 5.0(0.25) \mathrm{min}, 95 \% \mathrm{CI} 1.21$ to 2.39; $p<0.001)$ all decreased significantly in the cold.

NO TREATMENT VERSUS NIFEDIPINE (TABLE 4)

After two weeks' treatment with nifedipine there were significant increases in the exercise time to onset of angina (5.4v 7.8 (0.56) $\mathrm{min}, 95 \% \mathrm{CI} 1.08$ to 3.72 at $20^{\circ} \mathrm{C} ; \mathrm{p}<0.01 ; 3.5$ v $7.2(0.58) \mathrm{min}, 95 \%$ CI 2.33 to 5.07 at $\left.0^{\circ} \mathrm{C} ; \mathrm{p}<0.001\right)$ and to peak exercise $(6.8 \mathrm{v} 9.1$ (0.53) $\min , 95 \%$ CI 1.05 to 3.55 at $20^{\circ} \mathrm{C}$; p $<0.02$; 5.0 v 8.5(0.66) $\mathrm{min}, 95 \% \mathrm{CI} 1.94$ to 5.06 at $0^{\circ} \mathrm{C}$; $\mathrm{p}<$ $0.01)$. The time to $1 \mathrm{~mm}$ ST depression did not increase in the warm $\left(20^{\circ} \mathrm{C}\right)(4.5 v 5.8(1.3) \mathrm{min}, 95 \%$ CI -1.77 to 4.37 ; NS) but was significantly longer in the cold $\left(0^{\circ} \mathrm{C}\right)(3.1$ v $5.3(0.86) \mathrm{min}, 95 \% \mathrm{CI} 0.17$ to 4.23; $p<0.02$ ). At the end of the nifedipine 


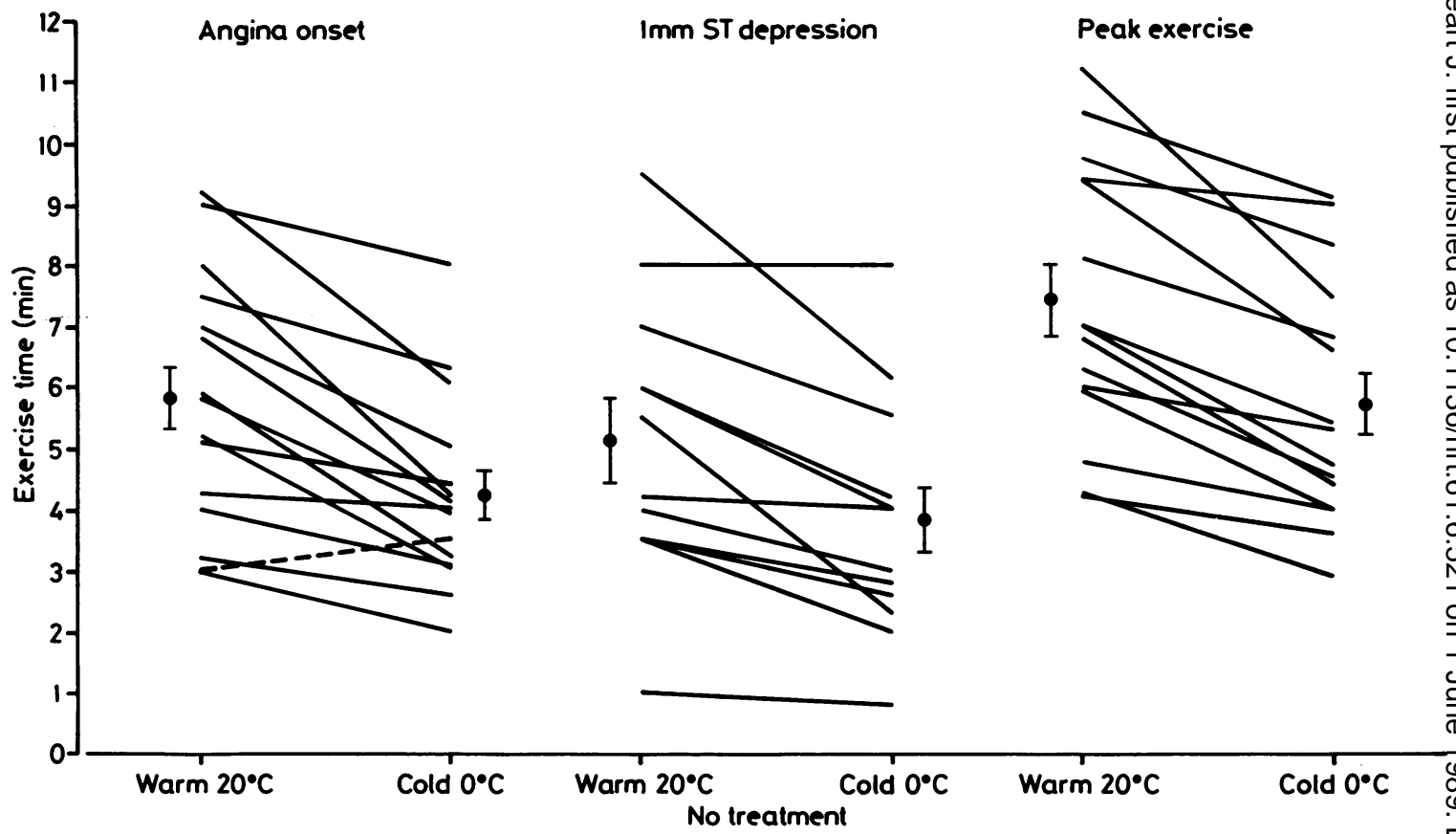

Figure Exercise time (in minutes) to onset of angina, to $1 \mathrm{~mm} S T$ depression, and to peak exercise in 15 patients on no antianginal medication at $20^{\circ} \mathrm{C}$ and at $0^{\circ} \mathrm{C}$. Mean time (SEM) to onset of angina $(p<0.001)$, to $1 \mathrm{~mm} S T$ depression $(p<0.005)$, and to peak exercise $(p<0.001)$ were all significantly reduced at $0^{\circ} \mathrm{C}$.

treatment period there was no cold intolerance in terms of time to peak exercise $\left(9.1 \mathrm{~min}\right.$ at $20^{\circ} \mathrm{C} v 8.5$ $\min$ at $0^{\circ} \mathrm{C}(0.42) \min , 95 \% \mathrm{CI}-0.4$ to $\left.1.6 ; \mathrm{NS}\right)$. The double product at the onset of angina, at $1 \mathrm{~mm} \mathrm{ST}$ depression, and at peak exercise was the same for both the warm and cold exercise tests on nifedipine, and the double product at these three stages was the same on nifedipine treatment as it was without treatment.

\section{NO TREATMENT VERSUS PROPRANOLOL (TABLE} 4)

After two weeks' treatment with propranolol there were significant increases in the exercise time to onset of angina (5.4v $7 \cdot 1(0.62) \mathrm{min}, 95 \% \mathrm{CI} 0.23$ to 3.17 at $20^{\circ} \mathrm{C} ; \mathrm{p}<0.05 ; 3.5$ v $6.2(0.66) \mathrm{min}, 95 \%$ CI 1.14 to 4.26 at $0^{\circ} \mathrm{C} ; \mathrm{p}<0.01$ ), to $1 \mathrm{~mm} \mathrm{ST}$ depression $4.5 v$ $6.5(0.68) \mathrm{min}, 95 \% \mathrm{CI} 0.39$ to 3.61 at $20^{\circ} \mathrm{C}$; p < $0.05 ; 3.1$ v $5.6(0.45) \mathrm{min}, 95 \% \mathrm{CI} 1.44$ to 3.56 at $0^{\circ} \mathrm{C}$; $\mathrm{p}<0.01)$, and to peak exercise $(6.8 v 8.9(0.52) \mathrm{min}$, $95 \%$ CI 0.87 to 3.33 at $20^{\circ} \mathrm{C}$; p < $0.01 ; 5.0$ v 7.8 (0.56) $\min , 95 \%$ CI 1.48 to 4.12 at $0^{\circ} \mathrm{C}$; p $<0.01$ ). Cold intolerance as judged by the time to peak exercise was still demonstrable at the end of the propranolol treatment period $(8.9$ v $7.8(0.23) \mathrm{min}$, $95 \%$ CI 0.56 to $1.64 ; \mathrm{p}<0.01$ ) although to a lesser extent than before treatment $\left(26.5 \% v 12 \cdot 3 \%\right.$ reduc- $\frac{\overrightarrow{\overrightarrow{0}}}{3}$ tion in exercise tolerance). The double product was the same at onset of angina, at $1 \mathrm{~mm} \mathrm{ST} \mathrm{depression}$ and at peak exercise in both the warm and cold exercise tests on propranolol. During treatment with propranolol, however, the double product was sig nificantly less at all points during exercise compared with the exercise tests on no treatment $(p<0.01)$.

NIFEDIPINE VERSUS PROPRANOLOL

There was no significant difference between the exercise time to onset of angina, to $1 \mathrm{~mm} S T$ depression, or to peak exercise on nifedipine or on proprạnolol. The double product, however, was significantly less at all points during exercise both in the warm and in the cold on propranolol as compared with nifedipine $(\mathrm{p}<0.01)$.

\section{Discussion}

A fall in the environmental temperature can have two effects on patients with ischaemic heart disease. The most common and immediately apparent is a con-而 siderable deterioration in their symptoms; Heberden $\frac{\text { }}{\bar{D}}$ first observed this in his classic description of angina pectoris. ${ }^{8}$ Over half of our patients with chronic 
Cold intolerance in patients with angina pectoris: effect of nifedipine and propranolol

Table 3 Exercise test data for the 15 patients exercised at $0^{\circ} \mathrm{C}$ and $20^{\circ} \mathrm{C}$ on no antianginal medication

\begin{tabular}{|c|c|c|c|c|c|c|c|c|c|c|c|c|c|c|c|c|}
\hline \multirow[b]{2}{*}{ No } & \multirow[b]{2}{*}{$W / C$} & \multicolumn{3}{|c|}{ At rest ${ }^{\star}$} & \multicolumn{4}{|c|}{ Angina onset } & \multicolumn{4}{|c|}{$1 \mathrm{~mm} S T$ depression } & \multicolumn{4}{|c|}{ Peak exercise } \\
\hline & & $H R$ & $B P$ & $\begin{array}{l}D P \\
\left(\times 10^{3}\right)\end{array}$ & Min & $H R$ & $B P$ & $\begin{array}{l}D P \\
\left(\times 10^{3}\right)\end{array}$ & Min & $H R$ & $B P$ & $\begin{array}{l}D P \\
\left(\times 10^{3}\right)\end{array}$ & Min & $H R$ & $B P$ & $\begin{array}{l}D P \\
\left(\times 10^{3}\right)\end{array}$ \\
\hline 1 & $\begin{array}{l}\mathrm{W} \\
\mathrm{C} \\
\mathrm{C}+1\end{array}$ & $\begin{array}{l}63 \\
62 \\
67\end{array}$ & $\begin{array}{r}100 \\
90 \\
104\end{array}$ & $\begin{array}{l}6.3 \\
5.6 \\
6.9\end{array}$ & $\begin{array}{l}4 \cdot 25 \\
4 \cdot 0\end{array}$ & $\begin{array}{l}110 \\
110\end{array}$ & $\begin{array}{l}110 \\
120\end{array}$ & $\begin{array}{l}12 \cdot 1 \\
13 \cdot 2\end{array}$ & $\begin{array}{l}4 \cdot 2 \\
4 \cdot 0\end{array}$ & $\begin{array}{l}110 \\
110\end{array}$ & $\begin{array}{l}120 \\
124\end{array}$ & $\begin{array}{l}13 \cdot 2 \\
13 \cdot 6\end{array}$ & $\begin{array}{l}6 \cdot 0 \\
5 \cdot 3\end{array}$ & $\begin{array}{l}112 \\
112\end{array}$ & $\begin{array}{l}112 \\
130\end{array}$ & $\begin{array}{l}12 \cdot 5 \\
14 \cdot 6\end{array}$ \\
\hline 2 & $\begin{array}{l}\text { W } \\
\mathrm{C} \\
\mathrm{C}+1\end{array}$ & $\begin{array}{l}96 \\
92 \\
96\end{array}$ & $\begin{array}{l}176 \\
176 \\
190\end{array}$ & $\begin{array}{l}16 \cdot 9 \\
16 \cdot 2 \\
18 \cdot 2\end{array}$ & $\begin{array}{l}5 \cdot 1 \\
4 \cdot 4\end{array}$ & $\begin{array}{l}130 \\
130\end{array}$ & $\begin{array}{l}204 \\
200\end{array}$ & $\begin{array}{l}26 \cdot 5 \\
26 \cdot 0\end{array}$ & $\begin{array}{l}3.5 \\
2.0\end{array}$ & $\begin{array}{l}122 \\
122\end{array}$ & $\begin{array}{l}190 \\
190\end{array}$ & $\begin{array}{l}23 \cdot 2 \\
23 \cdot 2\end{array}$ & $\begin{array}{l}8 \cdot 1 \\
6 \cdot 8\end{array}$ & $\begin{array}{l}148 \\
140\end{array}$ & $\begin{array}{l}216 \\
216\end{array}$ & $\begin{array}{l}31.9 \\
30 \cdot 2\end{array}$ \\
\hline 3 & $\begin{array}{l}\text { W } \\
C \\
C+1\end{array}$ & $\begin{array}{l}76 \\
64 \\
68\end{array}$ & $\begin{array}{l}146 \\
156 \\
170\end{array}$ & $\begin{array}{r}11.1 \\
9.9 \\
11.5\end{array}$ & $\begin{array}{l}5.8 \\
3.9\end{array}$ & $\begin{array}{l}108 \\
106\end{array}$ & $\begin{array}{l}160 \\
160\end{array}$ & $\begin{array}{l}17.3 \\
16.9\end{array}$ & $\begin{array}{l}6 \cdot 0 \\
4 \cdot 2\end{array}$ & $\begin{array}{l}108 \\
108\end{array}$ & $\begin{array}{l}160 \\
160\end{array}$ & $\begin{array}{l}17 \cdot 3 \\
17 \cdot 3\end{array}$ & $\begin{array}{l}6.25 \\
4.5\end{array}$ & $\begin{array}{l}105 \\
109\end{array}$ & $\begin{array}{l}166 \\
156\end{array}$ & $\begin{array}{l}17 \cdot 4 \\
17.0\end{array}$ \\
\hline 4 & $\begin{array}{l}\mathrm{W} \\
\mathrm{C} \\
\mathrm{C}+1\end{array}$ & $\begin{array}{r}88 \\
92 \\
106\end{array}$ & $\begin{array}{l}130 \\
120 \\
130\end{array}$ & $\begin{array}{l}11.4 \\
11.0 \\
13.8\end{array}$ & $\begin{array}{l}7 \cdot 5 \\
6 \cdot 3\end{array}$ & $\begin{array}{l}140 \\
152\end{array}$ & $\begin{array}{l}180 \\
180\end{array}$ & $\begin{array}{l}25 \cdot 2 \\
27 \cdot 3\end{array}$ & $\begin{array}{l}8 \cdot 0 \\
8.0\end{array}$ & $\begin{array}{l}148 \\
160\end{array}$ & $\begin{array}{l}180 \\
180\end{array}$ & $\begin{array}{l}26 \cdot 6 \\
28 \cdot 8\end{array}$ & $\begin{array}{r}10 \cdot 5 \\
9 \cdot 1\end{array}$ & $\begin{array}{l}175 \\
180\end{array}$ & $\begin{array}{l}200 \\
190\end{array}$ & $\begin{array}{l}35 \cdot 0 \\
34 \cdot 2\end{array}$ \\
\hline 5 & $\begin{array}{l}\mathrm{W} \\
\mathrm{C} \\
\mathrm{C}+1\end{array}$ & $\begin{array}{l}78 \\
82 \\
90\end{array}$ & $\begin{array}{l}154 \\
144 \\
170\end{array}$ & $\begin{array}{l}12.0 \\
11.8 \\
15.3\end{array}$ & $\begin{array}{l}9 \cdot 2 \\
6.0\end{array}$ & $\begin{array}{l}170 \\
170\end{array}$ & $\begin{array}{l}212 \\
210\end{array}$ & $\begin{array}{l}36 \cdot 0 \\
35.7\end{array}$ & $\begin{array}{l}9 \cdot 5 \\
6 \cdot 1\end{array}$ & $\begin{array}{l}170 \\
170\end{array}$ & $\begin{array}{l}212 \\
220\end{array}$ & $\begin{array}{l}36.0 \\
37 \cdot 4\end{array}$ & $\begin{array}{r}11 \cdot 2 \\
7 \cdot 4\end{array}$ & $\begin{array}{l}180 \\
170\end{array}$ & $\begin{array}{l}216 \\
222\end{array}$ & $\begin{array}{l}38.9 \\
37.7\end{array}$ \\
\hline $6+$ & $\begin{array}{l}\text { W } \\
C \\
C+1\end{array}$ & $\begin{array}{l}63 \\
74 \\
66\end{array}$ & $\begin{array}{l}120 \\
122 \\
136\end{array}$ & $\begin{array}{l}7.6 \\
9.0 \\
8.9\end{array}$ & $\begin{array}{l}9.0 \\
8.0\end{array}$ & $\begin{array}{l}160 \\
160\end{array}$ & $\begin{array}{l}160 \\
170\end{array}$ & $\begin{array}{l}25 \cdot 6 \\
27 \cdot 2\end{array}$ & - & - & - & $\overline{-}$ & $\begin{array}{l}9 \cdot 4 \\
9 \cdot 0\end{array}$ & $\begin{array}{l}160 \\
160\end{array}$ & $\begin{array}{l}160 \\
174\end{array}$ & $\begin{array}{l}25 \cdot 6 \\
27 \cdot 8\end{array}$ \\
\hline 7 & $\begin{array}{l}\text { W } \\
\text { C } \\
\text { C }+1\end{array}$ & $\begin{array}{l}64 \\
68 \\
70\end{array}$ & $\begin{array}{l}124 \\
110 \\
140\end{array}$ & $\begin{array}{l}7.9 \\
7.5 \\
9.8\end{array}$ & $\begin{array}{l}3 \cdot 2 \\
2 \cdot 6\end{array}$ & $\begin{array}{l}100 \\
112\end{array}$ & $\begin{array}{l}140 \\
150\end{array}$ & $\begin{array}{l}14.0 \\
16.8\end{array}$ & $\begin{array}{l}3.5 \\
2.6\end{array}$ & $\begin{array}{l}102 \\
112\end{array}$ & $\begin{array}{l}144 \\
150\end{array}$ & $\begin{array}{l}14 \cdot 7 \\
16 \cdot 8\end{array}$ & $\begin{array}{l}4.75 \\
4.0\end{array}$ & $\begin{array}{l}107 \\
120\end{array}$ & $\begin{array}{l}150 \\
142\end{array}$ & $\begin{array}{l}16.0 \\
17.0\end{array}$ \\
\hline 8 & $\begin{array}{l}\text { W } \\
\mathrm{C} \\
\mathrm{C}+1\end{array}$ & $\begin{array}{l}70 \\
74 \\
80\end{array}$ & $\begin{array}{l}130 \\
138 \\
166\end{array}$ & $\begin{array}{r}9 \cdot 1 \\
10 \cdot 2 \\
13 \cdot 2\end{array}$ & $\begin{array}{l}8 \cdot 0 \\
4 \cdot 2\end{array}$ & $\begin{array}{l}155 \\
146\end{array}$ & $\begin{array}{l}172 \\
180\end{array}$ & $\begin{array}{l}26 \cdot 7 \\
26 \cdot 2\end{array}$ & $\begin{array}{l}5 \cdot 5 \\
2 \cdot 3\end{array}$ & $\begin{array}{l}130 \\
124\end{array}$ & $\begin{array}{l}164 \\
174\end{array}$ & $\begin{array}{l}21 \cdot 3 \\
21 \cdot 6\end{array}$ & $\begin{array}{l}9 \cdot 4 \\
6 \cdot 6\end{array}$ & $\begin{array}{l}158 \\
150\end{array}$ & $\begin{array}{l}170 \\
176\end{array}$ & $\begin{array}{l}26.9 \\
26.4\end{array}$ \\
\hline 9 & $\begin{array}{l}\mathrm{W} \\
\mathrm{C} \\
\mathrm{C}+1\end{array}$ & $\begin{array}{l}68 \\
70 \\
72\end{array}$ & $\begin{array}{l}136 \\
120 \\
136\end{array}$ & $\begin{array}{l}9 \cdot 2 \\
8.4 \\
9 \cdot 7\end{array}$ & $\begin{array}{l}7 \cdot 0 \\
5 \cdot 0\end{array}$ & $\begin{array}{l}144 \\
120\end{array}$ & $\begin{array}{l}162 \\
176\end{array}$ & $\begin{array}{l}23 \cdot 3 \\
21 \cdot 1\end{array}$ & $\begin{array}{l}7 \cdot 0 \\
5 \cdot 5\end{array}$ & $\begin{array}{l}144 \\
120\end{array}$ & $\begin{array}{l}162 \\
176\end{array}$ & $\begin{array}{l}23 \cdot 3 \\
21 \cdot 1\end{array}$ & $\begin{array}{l}9 \cdot 75 \\
8 \cdot 3\end{array}$ & $\begin{array}{l}150 \\
140\end{array}$ & $\begin{array}{l}176 \\
180\end{array}$ & $\begin{array}{l}26 \cdot 4 \\
25 \cdot 2\end{array}$ \\
\hline 10 & $\begin{array}{l}\text { W } \\
\text { C } \\
\text { C+1 }\end{array}$ & $\begin{array}{l}89 \\
96 \\
96\end{array}$ & $\begin{array}{l}128 \\
144 \\
155\end{array}$ & $\begin{array}{l}11.4 \\
13.8 \\
14.9\end{array}$ & $\begin{array}{l}5.9 \\
3.2\end{array}$ & $\begin{array}{l}146 \\
130\end{array}$ & $\begin{array}{l}172 \\
174\end{array}$ & $\begin{array}{l}25 \cdot 1 \\
22 \cdot 6\end{array}$ & $\begin{array}{l}4 \cdot 0 \\
3 \cdot 0\end{array}$ & $\begin{array}{l}140 \\
130\end{array}$ & $\begin{array}{l}156 \\
174\end{array}$ & $\begin{array}{l}21 \cdot 8 \\
22.6\end{array}$ & $\begin{array}{l}7 \cdot 0 \\
5 \cdot 4\end{array}$ & $\begin{array}{l}150 \\
140\end{array}$ & $\begin{array}{l}176 \\
192\end{array}$ & $\begin{array}{l}26.4 \\
26.9\end{array}$ \\
\hline 11 & $\begin{array}{l}\text { W } \\
\mathrm{C} \\
\mathrm{C}+1\end{array}$ & $\begin{array}{l}82 \\
76 \\
80\end{array}$ & $\begin{array}{l}136 \\
138 \\
144\end{array}$ & $\begin{array}{l}11 \cdot 1 \\
10.5 \\
11.5\end{array}$ & $\begin{array}{l}4 \cdot 0 \\
3 \cdot 1\end{array}$ & $\begin{array}{l}106 \\
100\end{array}$ & $\begin{array}{l}160 \\
162\end{array}$ & $\begin{array}{l}16.9 \\
16.2\end{array}$ & $\begin{array}{l}1.0 \\
0.8\end{array}$ & $\begin{array}{l}94 \\
96\end{array}$ & $\begin{array}{l}150 \\
150\end{array}$ & $\begin{array}{l}14 \cdot 1 \\
14 \cdot 4\end{array}$ & $\begin{array}{l}5.9 \\
4.0\end{array}$ & $\begin{array}{l}104 \\
108\end{array}$ & $\begin{array}{l}172 \\
178\end{array}$ & $\begin{array}{l}17.9 \\
19.2\end{array}$ \\
\hline $12+$ & $\begin{array}{l}\text { W } \\
C \\
C+1\end{array}$ & $\begin{array}{l}94 \\
92 \\
90\end{array}$ & $\begin{array}{l}140 \\
150 \\
162\end{array}$ & $\begin{array}{l}13 \cdot 1 \\
13 \cdot 8 \\
14 \cdot 6\end{array}$ & $\begin{array}{l}3.0 \\
3.5\end{array}$ & $\begin{array}{l}148 \\
144\end{array}$ & $\begin{array}{l}196 \\
208\end{array}$ & $\begin{array}{l}29.0 \\
29.9\end{array}$ & - & - & - & - & $\begin{array}{l}4 \cdot 2 \\
3 \cdot 6\end{array}$ & $\begin{array}{l}150 \\
144\end{array}$ & $\begin{array}{l}212 \\
210\end{array}$ & $\begin{array}{l}31 \cdot 8 \\
30 \cdot 2\end{array}$ \\
\hline 13 & $\begin{array}{l}\text { W } \\
\mathrm{C} \\
\mathrm{C}+1\end{array}$ & $\begin{array}{l}104 \\
104 \\
108\end{array}$ & $\begin{array}{l}152 \\
160 \\
176\end{array}$ & $\begin{array}{l}15.8 \\
16.6 \\
19.0\end{array}$ & $\begin{array}{l}6 \cdot 8 \\
4 \cdot 1\end{array}$ & $\begin{array}{l}176 \\
180\end{array}$ & $\begin{array}{l}210 \\
210\end{array}$ & $\begin{array}{l}36 \cdot 9 \\
37 \cdot 8\end{array}$ & - & - & - & - & $\begin{array}{l}7 \cdot 0 \\
4 \cdot 7\end{array}$ & $\begin{array}{l}176 \\
180\end{array}$ & $\begin{array}{l}212 \\
212\end{array}$ & $\begin{array}{l}37 \cdot 3 \\
38 \cdot 2\end{array}$ \\
\hline 14 & $\begin{array}{l}\text { W } \\
\mathrm{C} \\
\mathrm{C}+1\end{array}$ & $\begin{array}{l}96 \\
80 \\
78\end{array}$ & $\begin{array}{l}118 \\
130 \\
136\end{array}$ & $\begin{array}{l}11 \cdot 3 \\
10 \cdot 4 \\
10 \cdot 6\end{array}$ & $\begin{array}{l}3 \cdot 0 \\
2 \cdot 0\end{array}$ & $\begin{array}{l}124 \\
106\end{array}$ & $\begin{array}{l}162 \\
160\end{array}$ & $\begin{array}{l}20 \cdot 1 \\
16 \cdot 9\end{array}$ & $\begin{array}{l}3.5 \\
2.8\end{array}$ & $\begin{array}{l}126 \\
112\end{array}$ & $\begin{array}{l}164 \\
160\end{array}$ & $\begin{array}{l}20.7 \\
17.9\end{array}$ & $\begin{array}{l}4 \cdot 25 \\
2.9\end{array}$ & $\begin{array}{l}120 \\
112\end{array}$ & $\begin{array}{l}166 \\
162\end{array}$ & $\begin{array}{l}19.9 \\
18 \cdot 1\end{array}$ \\
\hline 15 & $\begin{array}{l}\mathrm{W} \\
\mathrm{C} \\
\mathrm{C}+1\end{array}$ & $\begin{array}{l}66 \\
75 \\
78\end{array}$ & $\begin{array}{l}150 \\
146 \\
176\end{array}$ & $\begin{array}{r}9.9 \\
10.9 \\
13.7\end{array}$ & $\begin{array}{l}5 \cdot 2 \\
3 \cdot 0\end{array}$ & $\begin{array}{l}140 \\
140\end{array}$ & $\begin{array}{l}176 \\
180\end{array}$ & $\begin{array}{l}24 \cdot 6 \\
25 \cdot 2\end{array}$ & $\begin{array}{l}6 \cdot 0 \\
4 \cdot 0\end{array}$ & $\begin{array}{l}144 \\
144\end{array}$ & $\begin{array}{l}180 \\
182\end{array}$ & $\begin{array}{l}25.9 \\
26 \cdot 2\end{array}$ & $\begin{array}{l}6 \cdot 8 \\
4 \cdot 4\end{array}$ & $\begin{array}{l}150 \\
140\end{array}$ & $\begin{array}{l}182 \\
190\end{array}$ & $\begin{array}{l}27 \cdot 3 \\
26 \cdot 6\end{array}$ \\
\hline
\end{tabular}

*Values before entry into cold room $\left(0^{\circ} \mathrm{C}\right)$.

†No significant ST depression in three patients.

$\mathrm{HR}$, heart rate; BP, systolic blood pressure $(\mathrm{mm} \mathrm{Hg}) ; \mathrm{DP}$, double product of heart rate $\times$ blood pressure; W, warm $\left(20^{\circ} \mathrm{C}\right)$ exercise test; $\mathrm{C}$, cold $\left(0^{\circ} \mathrm{C}\right)$ exercise test; $\mathrm{C}+1=\mathrm{HR}, \mathrm{BP}, \mathrm{DP}$ after $1 \mathrm{~min}$ in cold room $\left(0^{\circ} \mathrm{C}\right)$ before starting exercise test.

stable angina pectoris commented that in cold weather not only did the frequency and severity of their symptoms increase but also that symptoms were more easily provoked. ${ }^{1}$ Cold weather also causes an overall increase in cardiovascular mortality. ${ }^{9}$

Several studies have examined the relation between environmental temperature and cardiovascular mortality. ${ }^{10-12}$ In 1966 Rose analysed the seasonal distribution of death and reinfarction in the Medical Research Council trial of long term anticoagulant treatment after acute myocardial infarction and showed that reinfarction was almost twice as common in December as in June and that the "winter excess of deaths in any particular year was very highly correlated with coldness". " $\mathrm{He}$ concluded that changes in temperature were responsible for most of the short term fluctuations in mortality from ischaemic heart disease. Further evidence from Canada confirmed the importance of a sudden drop in temperature in increasing deaths from ischaemic heart disease, especially when the cold stimulus was accompanied by exercise, such as clearing snow. ${ }^{13}$ In the Canadian study the daily rate of sudden death increased during "cold snaps" (defined as days when the temperature was at least $4.4^{\circ} \mathrm{C}$ lower than the previous day) and around the time of heavy snow- 
Table 4 Exercise test data (mean (SD)) for the eight patients in the pharmacological study. Data are presented for exercise testing before treatment, after two weeks on nifedipine, and after two weeks on propranolol

\begin{tabular}{|c|c|c|c|c|c|c|c|c|c|}
\hline & \multirow{2}{*}{\multicolumn{3}{|c|}{$\frac{\text { No treatment }}{\text { Time to: }}$}} & \multirow{2}{*}{\multicolumn{3}{|c|}{$\frac{\text { Nifedipine }}{\text { Time to: }}$}} & \multirow{2}{*}{\multicolumn{3}{|c|}{$\frac{\text { Propranolol }}{\text { Time to: }}$}} \\
\hline & & & & & & & & & \\
\hline & $\begin{array}{l}\text { AP onset } \\
(\text { min })\end{array}$ & $\operatorname{lmm}_{(\min )} S T \downarrow$ & $\begin{array}{l}\text { Peak } \\
\text { exercise } \\
(\text { min })\end{array}$ & $\begin{array}{l}\text { AP onset } \\
(\text { min })\end{array}$ & $\underset{(\min )}{1 \operatorname{mm}} S T \downarrow$ & $\begin{array}{l}\text { Peak } \\
\text { exercise } \\
(\text { min })\end{array}$ & $\begin{array}{l}\text { AP onset } \\
\text { (min) }\end{array}$ & $\underset{(\min )}{1 \operatorname{mm} S T \downarrow}$ & $\begin{array}{l}\text { Peak } \\
\text { exercise } \\
(\text { min })\end{array}$ \\
\hline $\begin{array}{l}\text { Warm }\left(20^{\circ} \mathrm{C}\right) \\
\text { exercise test }\end{array}$ & $\Gamma^{5.4(1.9)}$ & $\Gamma^{4 \cdot 5(2 \cdot 1)}$ & $\Gamma^{6 \cdot 8(2 \cdot 1)}$ & $\Gamma^{7 \cdot 8(2 \cdot 2)}$ & $5 \cdot 8(0.8)$ & $9 \cdot 1(2 \cdot 0)$ & $\Gamma^{7 \cdot 1(2 \cdot 1)}$ & $\Gamma^{6.5(1.6)}$ & $\Gamma^{8.9(2.4)}$ \\
\hline $\begin{array}{l}\text { Cold }\left(0^{\circ} \mathrm{C}\right) \\
\text { exercise test }\end{array}$ & $L_{3.5}(0.9)$ & $L_{3 \cdot 1(1 \cdot 6)}$ & $L_{5.0}(1.7)$ & $L_{7 \cdot 2(2 \cdot 2)}$ & $5 \cdot 3(1.8)$ & $8 \cdot 5(2 \cdot 3)$ & $L_{6} \cdot 2(2 \cdot 2)$ & $L_{5.6(1.9)}$ & $L_{7.8(2.6)}$ \\
\hline $\begin{array}{l}\text { Cold }\left(0^{\circ} \mathrm{C}\right) \\
\text { exercise test }\end{array}$ & $\mathrm{DP}\left(\times 10^{3}\right)$ & $\mathrm{DP}\left(\times 10^{3}\right)$ & $\mathrm{DP}\left(\times 10^{3}\right)$ & $\mathrm{DP}\left(\times 10^{3}\right)$ & $\mathrm{DP}\left(\times 10^{3}\right)$ & $\mathrm{DP}\left(\times 10^{3}\right)$ & $\mathrm{DP}\left(\times 10^{3}\right)$ & $\mathrm{DP}\left(\times 10^{3}\right)$ & $\left(\mathrm{DP} \times 10^{3}\right)$ \\
\hline \multirow{2}{*}{$\begin{array}{l}\text { Warm }\left(20^{\circ} \mathrm{C}\right) \\
\text { exercise test } \\
\text { Cold }\left(0^{\circ} \mathrm{C}\right) \\
\text { exercise test }\end{array}$} & $25 \cdot 3(6 \cdot 0)$ & $21 \cdot 2(3.9)$ & $26 \cdot 7(6 \cdot 1)$ & $25 \cdot 2(5 \cdot 5)$ & $21 \cdot 7(4 \cdot 1)$ & $27.8(4.9)$ & $16 \cdot 1(3 \cdot 6)$ & $16 \cdot 1(3 \cdot 2)$ & $17.5(4 \cdot 3)$ \\
\hline & $24 \cdot 5(7 \cdot 1)$ & $20 \cdot 6(4 \cdot 1)$ & $26 \cdot 3(6 \cdot 3)$ & $26 \cdot 0(3.5)$ & $22 \cdot 9(3 \cdot 7)$ & $27 \cdot 7(3 \cdot 6)$ & $16 \cdot 3(3 \cdot 4)$ & $15.9(3 \cdot 3)$ & $17 \cdot 5(4 \cdot 2)$ \\
\hline
\end{tabular}

${ }^{\star} \mathrm{p}<0.01 ;{ }^{\star \star} \mathrm{p}<0.05 ;{ }^{\star \star \star} \mathrm{p}<0.001 ;{ }^{\star \star \star \star} \mathrm{p}<0.02$

AP, angina.

falls, particularly in men under 65 years of age. The reasons for the increased mortality and the mechanisms by which it occurs are unknown and beyond the scope of our study, although in at least one study unaccustomed exertion seemed to play a part. ${ }^{13}$

Despite the frequency with which patients complain of deteriorating symptoms in winter, no studies have attempted to simulate the environmental conditions experienced by these patients on an average winter's day in Britain or have examined the effects of antianginal treatment. In part this is because it is difficult to obtain objective evidence of cold in- tolerance in individual patients. Two studies from Sweden have investigated certain aspects of the problem but at the much lower temperatures found in Sweden. ${ }^{214}$ Lassvik and Areskog studied 17 men with angina pectoris and cold intolerance who performed submaximal bicycle exercise tests at $20^{\circ} \mathrm{C}$ and $-10^{\circ} \mathrm{C} .{ }^{2}$ Despite their history of cold intolerance, the phenomenon could only be shown in $59 \%$ and the reduction in exercise tolerance was only about $10 \%$, a much smaller reduction than would be expected from their history. The failure to show the effect may have related to the fact that the laboratory procedure failed

Table 5 Exercise test data for the eight patients involved in the pharmacological study

\begin{tabular}{|c|c|c|c|c|c|c|c|c|c|c|c|c|c|c|c|}
\hline & & \multicolumn{7}{|c|}{ No treatment } & \multicolumn{7}{|c|}{ Nifedipine } \\
\hline & & \multirow{2}{*}{$\begin{array}{l}\text { Rest } \\
D P \\
\left(\times 10^{3}\right)\end{array}$} & \multicolumn{2}{|c|}{ AP onset } & \multicolumn{2}{|c|}{$1 \mathrm{~mm} S T \downarrow$} & \multicolumn{2}{|c|}{ Peak exercise } & \multirow{2}{*}{$\begin{array}{l}\text { Rest } \\
D P \\
\left(\times 10^{3}\right)\end{array}$} & \multicolumn{2}{|c|}{ AP onset } & \multicolumn{2}{|c|}{$1 \mathrm{~mm} S T \downarrow$} & \multicolumn{2}{|c|}{ Peak exercife } \\
\hline & & & $\min$ & $\begin{array}{l}D P \\
\left(\times 10^{3}\right)\end{array}$ & $\min$ & $\begin{array}{l}D P \\
\left(\times 10^{3}\right)\end{array}$ & $\min$ & $\begin{array}{l}D P \\
\left(\times 10^{3}\right)\end{array}$ & & $\min$ & $\begin{array}{l}D P \\
\left(\times 10^{3}\right)\end{array}$ & $\min$ & $\begin{array}{l}D P \\
\left(\times 10^{3}\right)\end{array}$ & $\min$ & $\left(\times \frac{p D}{=} 0^{3}\right)$ \\
\hline 1 & $\mathbf{W}$ & $9 \cdot 1$ & $8 \cdot 0$ & $26 \cdot 7$ & $5 \cdot 5$ & $21 \cdot 3$ & $9 \cdot 4$ & $26 \cdot 9$ & $15 \cdot 4$ & $10 \cdot 1$ & $33 \cdot 3$ & 5.5 & 26.5 & $11 \cdot 2$ & 33.90 \\
\hline & C & $13 \cdot 2$ & $4 \cdot 2$ & $26 \cdot 2$ & $2 \cdot 3$ & $21 \cdot 6$ & 6.6 & $26 \cdot 4$ & 13.9 & $9 \cdot 2$ & $28 \cdot 4$ & $4 \cdot 2$ & $22 \cdot 6$ & $10 \cdot 0$ & 2910 \\
\hline 2 & W & $\begin{array}{l}9.2 \\
9.7\end{array}$ & $\begin{array}{l}7.0 \\
5.0\end{array}$ & $\begin{array}{l}23 \cdot 3 \\
21 \cdot 1\end{array}$ & $\begin{array}{l}7 \cdot 0 \\
5 \cdot 5\end{array}$ & $\begin{array}{l}23 \cdot 3 \\
21 \cdot 1\end{array}$ & $\begin{array}{l}9 \cdot 75 \\
8.3\end{array}$ & $\begin{array}{l}26 \cdot 4 \\
25 \cdot 2\end{array}$ & $\begin{array}{l}8 \cdot 5 \\
0.5\end{array}$ & $9 \cdot 0$ & $26 \cdot 1$ & - & - & 9.9 & $30 \mathbb{ళ}$ \\
\hline 3 & $\widetilde{W}$ & $11 \cdot 4$ & $\begin{array}{l}5.0 \\
5.9\end{array}$ & $\begin{array}{l}21 \cdot 1 \\
25 \cdot 1\end{array}$ & $\begin{array}{l}5 \cdot 5 \\
4 \cdot 0\end{array}$ & $\begin{array}{l}21 \cdot 1 \\
21 \cdot 8\end{array}$ & $\begin{array}{l}8 \cdot 3 \\
7 \cdot 0\end{array}$ & $\begin{array}{l}23 \cdot 2 \\
26 \cdot 4\end{array}$ & $\begin{array}{r}9 \cdot 5 \\
11 \cdot 4\end{array}$ & $\begin{array}{l}8 \cdot 0 \\
9 \cdot 4\end{array}$ & $\begin{array}{l}25 \cdot 8 \\
24 \cdot 0\end{array}$ & $\overline{7 \cdot 0}$ & $\overline{20} \cdot 7$ & $\begin{array}{r}9 \cdot 6 \\
10.5\end{array}$ & 2905 \\
\hline & C & 14.9 & $3 \cdot 2$ & $22 \cdot 6$ & 3.0 & 22.6 & $5 \cdot 4$ & 26.9 & $14 \cdot 3$ & $\begin{array}{l}9.4 \\
9 \cdot 2\end{array}$ & $27 \cdot 8$ & $8 \cdot 0$ & $27 \cdot 3$ & 10.7 & $30 . \overline{1}$ \\
\hline 4 & $\mathbf{W}$ & $11 \cdot 1$ & $4 \cdot 0$ & 16.9 & $1 \cdot 0$ & $14 \cdot 1$ & 5.9 & $17 \cdot 9$ & $12 \cdot 8$ & $8 \cdot 5$ & $20 \cdot 0$ & $5 \cdot 6$ & $16 \cdot 6$ & $11 \cdot 2$ & 25 \\
\hline & C & $11 \cdot 5$ & $3 \cdot 1$ & $16 \cdot 2$ & 0.8 & $14 \cdot 4$ & $4 \cdot 0$ & $19 \cdot 2$ & $11 \cdot 1$ & $7 \cdot 7$ & $22 \cdot 7$ & $4 \cdot 0$ & $18 \cdot 3$ & $9 \cdot 8$ & 25 再 \\
\hline 5 & $\mathbf{W}$ & $13 \cdot 1$ & $3 \cdot 0$ & $29 \cdot 0$ & - & - & $4 \cdot 2$ & $31 \cdot 8$ & $12 \cdot 5$ & $7 \cdot 2$ & $25 \cdot 0$ & - & - & $7 \cdot 5$ & $25: 6$ \\
\hline & C & 14.6 & 3.5 & 29.9 & - & - & $3 \cdot 6$ & $30 \cdot 2$ & $13 \cdot 3$ & $6 \cdot 2$ & $26 \cdot 0$ & - & - & 7.0 & 260 \\
\hline 6 & $\mathbf{W}$ & $15 \cdot 8$ & $6 \cdot 8$ & 36.9 & - & - & $7 \cdot 0$ & $37 \cdot 3$ & $14 \cdot 7$ & $9 \cdot 0$ & $32 \cdot 3$ & - & - & 9.5 & $34 \sqrt{6}$ \\
\hline & C & 19.0 & $4 \cdot 1$ & $37 \cdot 8$ & - & $\overline{0}$ & $4 \cdot 7$ & $38 \cdot 2$ & $19 \cdot 5$ & $9 \cdot 0$ & $31 \cdot 0$ & - & - & $10 \cdot 0$ & $32 \bar{\phi}^{\circ}$ \\
\hline 7 & $\mathbf{W}$ & $11 \cdot 3$ & 3.0 & $20 \cdot 1$ & 3.5 & $20 \cdot 7$ & $4 \cdot 25$ & 19.9 & $11 \cdot 1$ & 4.0 & $17 \cdot 5$ & - & - & 6.9 & $20 \Omega$ \\
\hline & C & $10 \cdot 6$ & $2 \cdot 0$ & 16.9 & $2 \cdot 8$ & $17 \cdot 9$ & $2 \cdot 9$ & $18 \cdot 1$ & $12 \cdot 1$ & $3 \cdot 2$ & $19 \cdot 8$ & - & - & 4.0 & 21.9 \\
\hline 8 & $\begin{array}{l}\mathbf{W} \\
\mathbf{C}\end{array}$ & $\begin{array}{r}9.9 \\
13.7\end{array}$ & $\begin{array}{l}5 \cdot 2 \\
3 \cdot 0\end{array}$ & $\begin{array}{l}24 \cdot 6 \\
25 \cdot 2\end{array}$ & $\begin{array}{l}6 \cdot 0 \\
4 \cdot 0\end{array}$ & $\begin{array}{l}25 \cdot 9 \\
26 \cdot 2\end{array}$ & $\begin{array}{l}6 \cdot 8 \\
4 \cdot 4\end{array}$ & $\begin{array}{l}27 \cdot 3 \\
26 \cdot 6\end{array}$ & $\begin{array}{l}11 \cdot 2 \\
11 \cdot 7\end{array}$ & $\begin{array}{l}5 \cdot 0 \\
5 \cdot 2\end{array}$ & $\begin{array}{l}23 \cdot 0 \\
26 \cdot 7\end{array}$ & $\begin{array}{l}5 \cdot 0 \\
5.0\end{array}$ & $\begin{array}{l}22 \cdot 9 \\
23 \cdot 5\end{array}$ & $\begin{array}{l}6 \cdot 0 \\
6 \cdot 6\end{array}$ & $\begin{array}{l}24.7 \\
269\end{array}$ \\
\hline
\end{tabular}

$\mathrm{DP}$, double product of heart rate $\times$ blood pressure; $\mathrm{AP}$, angina; $\mathrm{W}$, warm exercise test $\left(20^{\circ} \mathrm{C}\right)$; $\mathrm{C}$, cold exercise test $\left(0^{\circ} \mathrm{C}\right):$ figure for $\mathrm{DP}$ at $\frac{0}{\mathrm{j}} \mathrm{is}$ DP after $1 \mathrm{~min}$ in cold room; -, no significant ST depression. 
to reproduce the real life situation, ignoring important factors such as clothing, wind, etc.

The two mechanisms invoked to account for the phenomenon of cold intolerance are reflex increases in peripheral vascular resistance producing increased cardiac work, ${ }^{3}$ and reflex increases in coronary vascular resistance producing reduced coronary perfusion. ${ }^{4}$ Published studies suggest that the effect on peripheral vascular resistance is more important. In normal subjects and patients with angina pectoris exposure to cold has been shown to result in peripheral vasoconstriction ${ }^{15}$ and a rise in blood pressure. ${ }^{16}$ Hayward et al showed that blowing cold air on to the face produced bradycardia and an increase in forearm vascular resistance, ${ }^{3}$ a response thought to be mediated via the vagus nerve. The same response, however, was not seen if the cold air was applied to the abdomen. Lassvik and Areskog provided further evidence of the importance of increased peripheral vascular resistance by showing that blood pressure in patients with angina pectoris was significantly higher throughout exercise in the cold. ${ }^{2}$ In the present study we showed that even a short exposure to cold (one minute before the start of the cold exercise test) is sufficient to increase blood pressure $(p<0.001)$ and the double product $(p<0.01)$ significantly, while heart rate remained unchanged. This is, almost certainly, related to a reflex increase in peripheral vascular resistance. Although an increase in cardiac output might produce similar findings this is unlikely to be the case because an earlier study showed regional deterioration in left ventricular function in patients with ischaemic heart disease on exposure to cold. ${ }^{17}$ At each minute stage of all of the cold exercise

\begin{tabular}{|c|c|c|c|c|c|c|}
\hline \multicolumn{7}{|c|}{ Propranolol } \\
\hline \multirow{2}{*}{$\begin{array}{l}\text { Rest } \\
D P \\
\left(\times 10^{3}\right)\end{array}$} & \multicolumn{2}{|c|}{$A P$ onset } & \multicolumn{2}{|c|}{$1 \mathrm{~mm} S T \downarrow$} & \multicolumn{2}{|c|}{ Peak exercise } \\
\hline & $\min$ & $\begin{array}{l}D P \\
\left(\times 10^{3}\right)\end{array}$ & $\min$ & $\begin{array}{l}D P \\
\left(\times 10^{3}\right)\end{array}$ & $\min$ & $\begin{array}{l}D P \\
\left(\times 10^{3}\right)\end{array}$ \\
\hline $\begin{array}{r}8.3 \\
9.7 \\
5.2 \\
6.3 \\
8.1 \\
9.5 \\
7.9 \\
7.6 \\
7.1 \\
7.4 \\
12.2 \\
12.0 \\
12.0 \\
10.8 \\
7.3 \\
9.6\end{array}$ & $\begin{array}{r}10.1 \\
8.2 \\
8.5 \\
8.5 \\
9.7 \\
9.2 \\
7.5 \\
6.0 \\
6.1 \\
4.3 \\
5.7 \\
4.6 \\
4.5 \\
3.1 \\
5.0 \\
5.4\end{array}$ & $\begin{array}{l}21.8 \\
19.3 \\
16.5 \\
17.0 \\
17.6 \\
19.0 \\
12.9 \\
11.2 \\
13.2 \\
13.6 \\
20.9 \\
20.9 \\
13.2 \\
13.3 \\
13.3 \\
16.0\end{array}$ & $\begin{array}{l}7 \cdot 0 \\
5.8 \\
7.0 \\
7.0 \\
8.0 \\
7 \cdot 2 \\
4.0 \\
3.5 \\
- \\
\overline{5.0} \\
3.0 \\
\overline{-} \\
\overline{8.0} \\
7.3\end{array}$ & $\begin{array}{l}17.6 \\
15.8 \\
14.6 \\
15.2 \\
16.6 \\
17.9 \\
11 \cdot 1 \\
10.4 \\
\overline{-} \\
20.7 \\
20.3 \\
\overline{16} \\
16.4 \\
16.0\end{array}$ & $\begin{array}{c}13.3 \\
12.2 \\
10.0 \\
9.6 \\
10.2 \\
9.4 \\
9.5 \\
7.4 \\
7.0 \\
5.3 \\
7.25 \\
6.8 \\
5.5 \\
3.75 \\
8.3 \\
7.7\end{array}$ & $\begin{array}{l}25.0 \\
24.6 \\
18.5 \\
17.3 \\
17.6 \\
18.7 \\
13.2 \\
12.7 \\
14.3 \\
15.3 \\
21.9 \\
21.9 \\
12.6 \\
13.1 \\
17.3 \\
16.0\end{array}$ \\
\hline
\end{tabular}

tests both blood pressure and double product were significantly greater than values attained during the warm exercise tests. Blood pressure and double product at the onset of angina, at $1 \mathrm{~mm} \mathrm{ST} \mathrm{depres-}$ sion, and at peak exercise were the same during the warm and cold exercise tests (table 3 ) although these values were achieved much earlier in the cold. The overall reduction in exercise tolerance in these patients is therefore related to increased cardiac work at any particular stage of the cold exercise tests as measured by the double product, which is a good index of myocardial oxygen consumption. ${ }^{18}$

The importance of coronary vascular resistance in determining cold intolerance is more difficult to assess because much of the work on coronary vascular resistance used a painful, non-physiological stimulus, the cold pressor test. ${ }^{19}{ }^{20}$ Nevertheless, this method showed an increase in coronary vascular resistance during cold stimulation, which was inhibited by nifedipine. ${ }^{21} 22$ This effect is relevant to the treatment of coronary artery spasm.

In considering treatment for patients with cold intolerance it is helpful to remember the mechanisms of action of commonly used antianginal agents. The $\beta$ blockers, particularly non-cardioselective ones such as propranolol, increase peripheral vascular resistance by leaving peripheral vasoconstrictor forces unopposed, and they also increase coronary vascular resistance, thus reducing coronary perfusion. ${ }^{6}$ Despite these effects some recommend an increased dose in winter ${ }^{23}$ Calcium antagonists such as nifedipine, on the other hand, act mainly on vascular smooth muscle ${ }^{7}$ both in peripheral arterioles where they reduce peripheral vascular resistance and in coronary arteries where they produce dilatation and an increase in coronary blood flow and oxygen to the myocardium. These latter agents may, therefore, have advantages over $\beta$ blockers in the treatment of cold intolerant patients with angina.

We showed significant increases in exercise tolerance in both the warm and the cold when patients were treated with propranolol or nifedipine, and there was no significant difference in overall exercise tolerance on either drug. Whereas we were unable to show cold intolerance in patients treated with nifedipine it was still evident with propranolol, albeit to a lesser extent than when no treatment was given. We did not investigate the value of nitrates in such patients, but a small study from Norway of bicycle ergometry found that when glyceryl trinitrate was given to patients before they cycled the work capacity in the cold $\left(-17^{\circ} \mathrm{C}\right)$ increased to levels obtained at room temperature $\left(18^{\circ} \mathrm{C}\right)$ and that this was mainly due to a decrease in the systolic blood pressure during cold exercise on treatment. ${ }^{24}$ One explanation for the reduced systolic blood pressure 
would be a decrease in peripheral vascular resistance mediated by nitrate induced vasodilatation. Nitrates, however, are important venodilators and this action, by reducing preload, may lead to a reduction in systolic blood pressure by reducing cardiac output.

In conclusion, therefore, the phenomenon of cold intolerance can be shown at $0^{\circ} \mathrm{C}$ by symptom limited treadmill exercise testing, and the haemodynamic data obtained suggest that increases in peripheral vascular resistance are the major determinant of reduced exercise tolerance. There is little difference between nifedipine and propranolol but present evidence suggests that increased doses of calcium antagonists or nitrates would be useful in cold intolerant patients in the winter.

We thank the British Heart Foundation for providing the cold room, the National Heart Research Fund who supported IP, the Northern Regional Health Authority who supported REB, and Bayer Pharmaceuticals who supported CA.

\section{References}

1 Hall RJC, Bullock RE, Albers C. The effect of cold on patients with angina pectoris-a review. Postgrad Med J 1983;59(suppl 2):55-7.

2 Lassvik CT, Areskog N-H. Angina in cold environment. Reactions to exercise. Br Heart $J$ 1979;42: 396-401.

3 Hayward JM, Holmes WF, Gooden BA. Cardiovascular responses in man to a stream of cold air. Cardiovasc Res 1976;10:691-6.

4 Gunther S, Green L, Muller JE, Mudge GH, Grossman W. Inappropriate coronary vasoconstriction in patients with coronary artery disease: a role for nifedipine? Am J Cardiol 1979;44:793-7.

5 Feldman RL, Whittle JL, Pepine CJ, Conti CR. Regional coronary angiographic observations during cold stimulation in patients with exertional chest pain: comparison of diameter responses in normal and fixed stenotic vessels. Am Heart J 1981;102:822-30.

6 Gunnells JC. Treatment of systemic hypertension. In: Hurst JW, ed. The heart. 5th ed. New York: McGraw-Hill, 1982:1196-220.

7 Stone DL, Stephens JD, Banim SO. Coronary haemodynamic effects of nifedipine: comparison with glyceryl trinitrate. Br Heart J 1983;49:442-6.

8 Heberden W. Some account of a disorder of the breast. Medical Transactions of the Royal College of Physicians of London 1772;2:59-67.

9 Dunnigan MG, Harland WA. Seasonal incidence and mortality of ischaemic heart disease. Lancet 1970 ; ii:793-7.

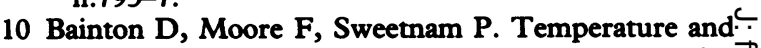
deaths from ischaemic heart disease. $B r J$ Prev Soc $\overrightarrow{\bar{N}}$ Med 1977;31:49-53.

11 Rose G. Cold weather and ischaemic heart disease. $B r \bar{C}$ Prev Soc Med 1966;20:97-100.

12 Baker-Blocker A. Winter weather and cardiovascular $\bar{T}$ mortality in Minneapolis-St. Paul. Am J Public Health 1982;72:261-5.

13 Anderson TW, Rochard C. Cold snaps, snowfall and sudden death from ischaemic heart disease. Can Med. Assoc J 1979;121:1580-3.

14 Backman C, Holm S, Linderholm H. Reaction to cold of patients with coronary insufficiency. Ups J Med Sct 1979;84:181-7.

15 Epstein SE, Stampfer M, Beiser GD, Goldstein RE, कृ Braunwald E. Effects of a reduction in environmentalos temperature on the circulatory response to exercise in man. Implications concerning angina pectoris. $N$ Eng $I$ J Med 1969;280:7-11.

16 Brennan PJ, Greenberg G, Miall W, Thompson SG.. Seasonal variation in arterial blood pressure. $\mathrm{Br} M e d c$ $J$ 1982;285:919-23.

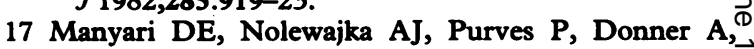
Kostuk WJ. Comparative value of the cold pressore test and supine bicycle exercise to detect subjects witho coronary artery disease using radionuclide ventri culography. Circulation 1982;65:571-9.

18 Gobel FL, Nordstrom LA, Nelson RR, Jorgensen CR, Wang Y. The rate-pressure product as an index of myocardial oxygen consumption during exercise in patients with angina pectoris. Circulation 1978; 57:549-56.

19 Raizner AE, Chahine RA, Ishimori T, et al. Provocation of coronary artery spasm by the cold pressor test. Haemodynamic, arteriographic and quantitative angiographic observations. Circulation 1980;62: 925-32.

20 Malacoff RF, Mudge GH, Holman BL, Idoine J Bifolck L, Cohn PF. Effect of the cold pressor test on regional myocardial blood flow in patients with coronary artery disease. Am Heart J 1983;106:78-84.

21 De Servi S, Mussini A, Specchia G, et al. Effects of nifedipine on coronary blood flow and coronary resistance during cold pressor test and ischaemic exercise in patients with coronary artery disease. EurD Heart J 1980;1(suppl B):43-7.

22 Goldhaber SZ, White MD, Holman BL, et al. Preven tion by nifedipine of cold pressor induced decrease in left ventricular ejection fraction. $\mathrm{J} \mathrm{Am}$ Coll Cardiol 1983;1:1512-7.

23 Anonymous. Cold and the heart. Br Med J 1974;iii:430. 24 Stanghelle JK, Nilsson S. Angina pectoris and the cold.Int Rehabil Med 1983;5:189-91. 